\title{
On Sufficient Conditions for the Existence of Stable Matchings with Contracts
}

\author{
Jun Zhang*
}

June 12, 2016

\begin{abstract}
We introduce two new sufficient conditions for the existence of stable outcomes in many-to-one matching with contracts. The conditions subsume the observable substitutability of Hatfield et al. (2015) and the substitutable completability of Hatfield and Kominers (2016) as special cases. We also prove that unilaterally substitutability and irrelevance of rejected contracts imply substitutable completability.
\end{abstract}

Keywords: Many-to-one Matching, Stability, Substitutability, Irrelevance of Rejected Contracts

JEL Classification: C62, C78, D78

*Division of Humanities and Social Sciences, California Institute of Technology. Address: 1200 East California Blvd, MC 228-77, Pasadena, CA 91125, USA. Email: jzzhang@caltech.edu. I thank Federico Echenique for guidance, and the editor and an anonymous referee for comments. All errors are mine. 


\section{Introduction}

Recently there are two advances in expanding the frontier of sufficient conditions for the existence of stable outcomes in the many-to-one matching with contracts model. One is achieved by Hatfield and Kominers (2016) who propose a condition called substitutable completability. Simply speaking, a choice function is substitutably completable if it has a completion in the many-to-many framework that satisfies substitutability (Hatfield and Milgrom, 2005). Hatfield and Kominers prove that if the choice functions of all firms have substitutable completions that satisfy irrelevance of rejected contracts (IRC; Aygün and Sönmez, 2013), then the worker-proposing cumulative offer algorithm can find stable outcomes.

The other advance is achieved by Hatfield et al. (2015). They propose the notion of observable offer processes, which, simply speaking, are the sequences of contracts that can appear in the worker-proposing cumulative offer algorithm. They call a firm's choice function observably substitutable if it satisfies substitutability on observable offer processes and IRC. They prove that if all firms have observably substitutable choice functions, the worker-proposing cumulative offer algorithm can find stable outcomes.

Before this note it is not clear how these two advances are related. In particular, even though a choice function has a substitutable completion that satisfies IRC, the choice function itself may not satisfy IRC. So it is not observably substitutable. On the other hand, Hatfield et al. show that an observably substitutable choice function may not have a substitutable completion. So the two advances seem independent of each other.

In this note we prove that if a choice function has a substitutable completion that satisfies IRC, then it must satisfy both substitutability and IRC on its observable offer processes. We call choice functions having this property weakly observably substitutable. We prove that weakly observable substitutability is sufficient for stable outcomes to exist. Since this condition also subsumes observable substitutability, the above two advances are unified in this note.

Inspired by the substitutable completion idea we conjecture that if all firms' choice functions have weakly observably substitutable completions, then stable outcomes may also exist. Interestingly, we find that a choice function has a completion that is weakly observably substitutable if and only if the choice function is weakly observable substitutable by itself. So the conjecture is indeed correct. This also implies that we cannot 
extend the substitutable completion idea beyond weakly observable substitutability.

We are also interested in the relation between unilateral substitutability introduced by Hatfield and Kojima (2010) and substitutable completability. Hatfield et al. show that unilateral substitutability is a special case of observable substitutability. In this note we constructively prove that if a choice function satisfies unilateral substitutability and IRC, it must have a substitutable completion. This result is more general than that of Kadam (2014) since he only proves the result for choice functions derived from preferences.

In the literature there are multiple sufficient conditions for the existence of stable outcomes in the many-to-one matching with contracts model. In particular, Hatfield et al propose another sufficient condition called observable substitutability across workers, which is weaker than observable substitutability. In this note we similarly define weakly observable substitutability across workers by requiring IRC hold only on observable offer processes. We prove that it is also a sufficient condition for stable outcomes to exist. In the following graph we summarize the relations between multiple sufficient conditions. It also clarifies our contribution in this note. In the graph "condition a $\rightarrow$ condition b" means that condition a implies condition b.

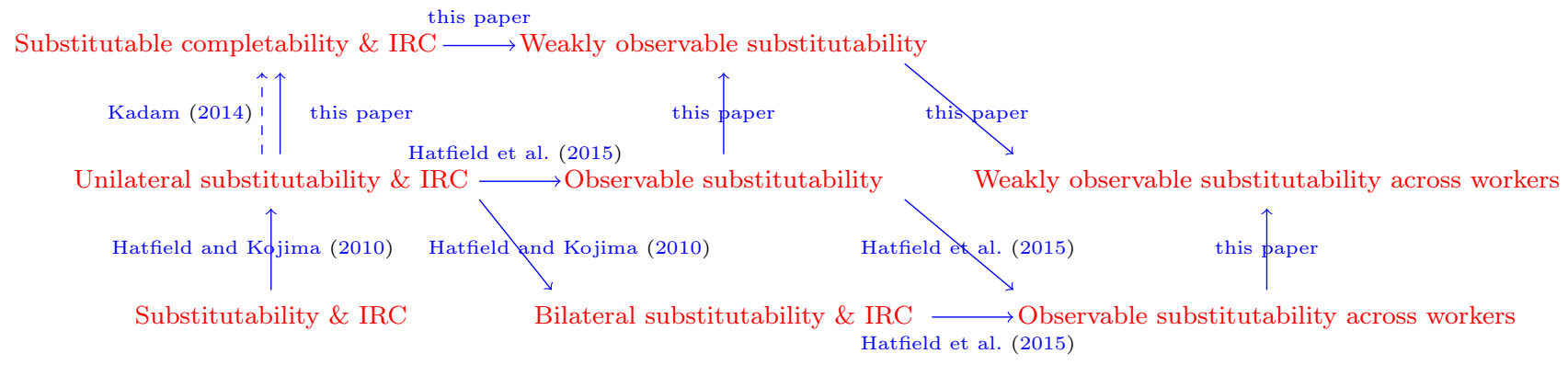

Figure 1: Relations between multiple sufficient conditions.

In Section 2 we define the standard many-to-one matching with contracts model. In Section 3 we define weakly observable substitutability and weakly observable substitutability across workers and prove that they are sufficient conditions. In Section 4 we prove that substitutable completability and IRC imply weakly observable substitutability. In Section 5 we prove that unilateral substitutability and IRC imply substitutable completability. In Section 5 we have some discussions. 


\section{Model}

We introduce the standard many-to-one matching with contracts model. There are a finite set $F$ of firms and a finite set $W$ of workers, with $I \equiv F \cup W$ being the set of all agents. Firms sign contracts with workers, but each worker can at most one contract. Let $X$ be the finite set of all contracts. For each contract $x \in X, w_{x}$ and $f_{x}$ are respectively the worker and the firm involved in $x$. For any subset of contracts $Z \subseteq X, Z_{i}$ is the set of contracts in $Z$ that involve any agent $i \in I, F_{Z}$ is the set of firms involved in $Z, W_{Z}$ is the set of workers involved in $Z$, and $I_{Z} \equiv F_{Z} \cup W_{Z}$ is the set of all agents involved in $Z$. A subset of contracts $A \subseteq X$ is called an outcome, and it is feasible if $\left|A_{w}\right| \leq 1$ for all $w \in W$.

Each worker $w$ has a strict preference relation $\succ_{w}$ over $X_{w} \cup\{\emptyset\}$, the set of all contracts involving $w$ and being unemployed. Every preference relation $\succ_{w}$ induces a choice function $C_{w}$ such that $C_{w}(Z) \equiv \arg \max _{x \in Z} \succ_{w}$ for all $Z \subseteq X_{w} \cup\{\emptyset\}$. Each firm $f \in F$ has a choice function $C_{f}$ such that for any $Z \subseteq X, C_{f}(Z) \subseteq Z_{f}$ contains at most one contract with each worker. In the terminology of Kominers (2012) we call such choice functions unitary. Let $C_{W} \equiv\left\{C_{w}\right\}_{w \in W}$ and $C_{F} \equiv\left\{C_{f}\right\}_{f \in F}$. The rejection function of each agent $i \in I$ is denoted by $R_{i}$ such that $R_{i}(Z) \equiv Z_{i} \backslash C_{i}(Z)$ for all $Z \subseteq X$. For all $Z \subseteq X$ and all $f \in F$, we use $C_{f}^{W}(Z)$ to denote the set of workers involved in $C_{f}(Z)$.

An outcome $A \subseteq X$ is individually rational if for all $i \in I, C_{i}(A)=A_{i}$. So an individually rational outcome must be feasible. An outcome $A$ is blocked by a set of contracts $Z \subseteq X$ if $Z \cap A=\emptyset$ and for all $i \in I_{Z}, Z_{i} \subseteq C_{i}(A \cup Z)$. An outcome $A$ is stable if it is individually rational and unblocked.

There are two conditions of choice functions that are important in matching with contracts: substitutability and irrevelence of rejected contracts (Hatfield and Milgrom, 2005; Aygün and Sönmez, 2013). If all firms' choice functions satisfy both conditions, then stable outcomes exist.

Definition 1. A firm's choice function $C_{f}$ is substitutable if for all $Z^{\prime} \subseteq Z \subseteq X$, $R_{f}\left(Z^{\prime}\right) \backslash R_{f}(Z)=\emptyset$.

Definition 2. A firm's choice function $C_{f}$ satisfies irrelevance of rejected contracts if for all $x \in Z \subseteq X$, if $x \notin C_{f}(Z)$, then $C_{f}(Z)=C_{f}(Z \backslash\{x\})$.

In next section we will propose two new conditions that are weaker than substitutabil- 
ity and IRC but are still sufficient for stable outcomes to exist. We will use the workerproposing cumulative offer $(\mathrm{COM})$ algorithm to prove the existence of stable outcomes. The formal definition of COM is below.

The worker-proposing cumulative offer algorithm:

- Step 0: Choose an arbitrary ordering $\triangleright_{W}$ of all workers. Initialize the set of cumulative offers that firms have received by step 0 as $A^{0} \equiv \emptyset$.

- Step $t \geq 1$ : Consider the set of workers who want to make an offer at step $t$ :

$$
W^{t}=\left\{w \in W: w \notin C_{F}^{W}\left(A^{t-1}\right), x_{w}^{t} \equiv \arg \max _{x \in X_{w} \cup\{\emptyset\} \backslash A^{t-1}} \succ_{w} \neq \emptyset\right\}
$$

If $W^{t} \neq \emptyset$, let the worker $w \in W^{t}$ who is ranked highest in $\triangleright_{W}$ makes the offer $x_{w}^{t}$. Then let $A^{t} \equiv A^{t-1} \cup\left\{x_{w}^{t}\right\}$. So $C_{F}\left(A^{t}\right) \equiv \cup_{f \in F} C_{f}\left(A_{f}^{t}\right)$ is the set of accepted contracts.

If $W^{t}=\emptyset$, then the algorithm terminates and the outcome is $C_{F}\left(A^{t-1}\right)$.

\section{Weakly Observable Substitutability (Across Work- ers)}

In this section we propose the notions of weakly observable substitutability and weakly observable substitutability across workers. To define them we first introduce some notions.

An offer process for firm $f$ is a finite sequence of distinct contracts $\mathbf{x}=\left(x^{1}, \cdots, x^{M}\right)$ such that for all $m=1, \cdots, M, x^{m} \in X_{f}$. For all $m=1, \cdots, M$, we denote $\mathbf{x}^{m} \equiv$ $\left(x^{1}, \cdots, x^{m}\right)$ and call it a subprocess of $\mathbf{x}$. We call $\mathbf{x}$ observable if for all $m=1, \cdots, M$, $w_{x^{m}} \notin C_{f}^{W}\left(\left\{x^{1}, \cdots, x^{m-1}\right\}\right)$. That is, the worker involved in $x^{m}$ is different from all workers involved in $C_{f}\left(\left\{x^{1}, \cdots, x^{m-1}\right\}\right)$.

A firm's choice function $C_{f}$ satisfies no observable violation of substitutability (NOVS) if for any two observable offer processes $\mathbf{x}, \mathbf{x}^{\prime}$ for $f$ such that $\mathbf{x}^{\prime}$ is a subprocess of $\mathbf{x}$, $R_{f}\left(\mathbf{x}^{\prime}\right) \backslash R_{f}(\mathbf{x})=\emptyset .{ }^{1}$ In a weaker notion $C_{f}$ satisfies no substantial violation of substitutability (NSVS) if for any two observable offer processes $\mathbf{x}, \mathbf{x}^{\prime}$ for $f$ such that $\mathbf{x}^{\prime}$ is a

\footnotetext{
${ }^{1}$ If $\mathbf{x}=\left(x^{1}, \cdots, x^{M}\right)$, we use $R_{f}(\mathbf{x})$ to denote $R_{f}\left(\left\{x^{1}, \cdots, x^{M}\right\}\right)$. In the original definition of Hatfield et al. (2015), their "if" condition is stated as "for any observable offer process $\left(x^{1}, \cdots, x^{M}\right)$ for $f$, $R_{f}\left(\left\{x^{1}, \cdots, x^{M-1}\right\}\right) \backslash R_{f}\left(\left\{x^{1}, \cdots, x^{M}\right\}\right)=\emptyset$." This condition is equivalent to the one in this note.
} 
subprocess of $\mathbf{x}$, if $x \in R_{f}\left(\mathbf{x}^{\prime}\right) \backslash R_{f}(\mathbf{x}) \neq \emptyset$, then $w_{x} \in C_{f}^{W}\left(\mathbf{x}^{\prime}\right)$. Then $C_{f}$ is observably substitutable if it satisfies NOVS and IRC, and $C_{f}$ is observably substitutable across workers if it satisfies NSVS and IRC.

Now we define a weak version of IRC which only requires IRC hold on observable offer processes.

Definition 3. A firm's choice function $C_{f}$ satisfies observable IRC if for all observable offer process $\boldsymbol{x}$ for $f$ and all $Z \subseteq R_{f}(\boldsymbol{x}), C_{f}(\boldsymbol{x})=C_{f}(\boldsymbol{x} \backslash Z)$.

A choice function must satisfy IRC if it is derived from a strict preference relation. In other words, if a choice function satisfies observable $I R C$ but not $I R C$, it cannot be derived from a strict preference relation.

Then we define the weaker versions of observable substitutability and observable substitutability across workers by replacing IRC with observable IRC.

Definition 4. A firm's choice function $C_{f}$ is weakly observably substitutable if it satifies NOVS and observable IRC.

Definition 5. A firm's choice function $C_{f}$ is weakly observably substitutable across workers if it satifies NSVS and observable IRC.

Note that weakly observable substitutability across workers is weaker than weakly observable substitutability. In the following we prove the existence of stable outcomes when the choice functions of all firms are weakly observably substitutable across workers. The proof is almost same as that of Hatfield et al.

Theorem 1. If the choice function of each firm is weakly observably substitutable across workers, then the outcome of the worker-proposing cumulative offer algorithm is stable.

Proof. Let $T$ be the last step of COM, then $A^{T}$ is the set of cumulative offers received by all firms. Let $Y=C_{F}\left(A^{T}\right) \equiv \cup_{f \in F} C_{f}\left(A_{f}^{T}\right)$, then $Y$ is the outcome of COM. So we need to prove that $Y$ is stable.

First, we prove that $Y$ is feasible. Suppose the contrary that there exist two contracts $x, x^{\prime} \in Y$ such that $w_{x}=w_{x^{\prime}}=w$ and without loss of generality $x \succ_{w} x^{\prime}$. So $w$ must propose $x$ earlier than proposing $x^{\prime}$. Suppose $w$ proposes $x^{\prime}$ at step $t$, then it must be that $x \in R_{f_{x}}\left(A_{f_{x}}^{t-1}\right)$. That is, $x$ is not accepted at the end of step $t-1$. However, $x \in Y$ implies that $x \notin R_{f_{x}}\left(A_{f_{x}}^{T}\right)$. Since $A_{f_{x}}^{t-1}$ is a subprocess of $A_{f_{x}}^{T}$ and $C_{f_{x}}$ satisfies 
NSVS, $w \in C_{f_{x}}^{W}\left(A_{f_{x}}^{t-1}\right)$. That is, there exists a contract $x^{\prime \prime} \in X$ such that $w_{x^{\prime \prime}}=w$ and $x^{\prime \prime} \in C_{f_{x}}\left(A_{f_{x}}^{t-1}\right)$. But this implies that $w$ should not make an offer at step $t$, which is a contradiction.

Second, we prove that $Y$ is individually rational. Since all workers must propose only acceptable contracts to firms, every contract in $Y$ is acceptable to the relevant worker. Since $Y$ is also feasible, it must be that for every $w, C_{w}(Y)=Y_{w}$. By construction $Y$ is individually rational for firms.

Lastly, we prove that $Y$ is unblocked. Consider any set $Z \subseteq X$ such that $Z \cap Y=\emptyset$ and $Z_{w} \subseteq C_{w}(Y \cup Z)$ for all $w \in W_{Z}$. For all $w \in W_{Z}, Z_{w}$ must be a single contract and $Z_{w} \succ_{w} Y_{w}$. So $w$ must propose $Z_{w}$ at some earlier step and is rejected before step $T$. Hence, $Z_{w} \in R_{f_{Z_{w}}}\left(A_{f_{Z_{w}}}^{T}\right) \subseteq A_{f_{Z_{w}}}^{T}$. Since $A_{f_{Z_{w}}}^{T}$ is an observable offer process for $f_{Z_{w}}$ and $C_{f_{Z_{w}}}$ satisfies observable IRC, $Y_{f_{Z_{w}}}=C_{f_{Z_{w}}}\left(A_{f_{Z_{w}}}^{T}\right)=C_{f_{Z_{w}}}\left(Y_{f_{Z_{w}}} \cup R_{f_{Z_{w}}}\left(A_{f_{Z_{w}}}^{T}\right)\right)=$ $C_{f_{Z_{w}}}\left(Y_{f_{Y_{w}}} \cup Z_{f_{Z_{w}}}\right)$. So $Z$ is not a blocking set.

\section{Substitutable Completability and IRC Imply Weakly Observable Substitutability}

Hatfield and Kominers (2016) have an innovative observation that although the choice functions of some firms are not substitutable, the firms may have underlying "substitutable preferences" which cannot be expressed in the many-to-one framework.

Definition 6. $\bar{C}_{f}$ is a completion of $C_{f}$ if for all $Z \subseteq X$, either $\bar{C}_{f}(Z)=C_{f}(Z)$ or there exists distinct $z, z^{\prime} \in \bar{C}_{f}(Z)$ such that $w_{z}=w_{z^{\prime}}$. Then $C_{f}$ is substitutably completable if it has a substitutable completion.

Hatfield and Kominers prove that if the choice functions of all firms have substitutable completions that satisfy IRC, then stable outcomes exist. However, although the substitutable completion of a choice function satisfies IRC, the choice function itself may not satisfy IRC. We provide a simple example to illustrate this fact.

Example 1. A firm $f$ has a choice function $C_{f}$ shown in Table 1. $w_{x}=w_{\hat{x}}=w_{1}$ and $w_{y}=w_{2} . \bar{C}_{f}$ is a completion of $C_{f}$.

Here $(x, \hat{x})$ and $(x, \hat{x}, y)$ are unobservable offer processes. $\bar{C}_{f}$ is substitutable and satisfies IRC. However, $C_{f}$ does not satisfy IRC since $\hat{x} \notin C_{f}(\{x, \hat{x}, y\})$ but $C_{f}(\{x, \hat{x}, y\})=$ 


\begin{tabular}{|c|c|c|}
\hline offer set $Z$ & $C_{f}(Z)$ & $\bar{C}_{f}(Z)$ \\
\hline$\{a\}: \forall a \in\{x, \hat{x}, y\}$ & $\{a\}$ & $\{a\}$ \\
\hline$\{x, y\}$ & $\{x, y\}$ & $\{x, y\}$ \\
\hline$\underline{\{x, \hat{x}\}}$ & $\underline{\{x\}}$ & $\underline{\{x, \hat{x}\}}$ \\
\hline$\{\hat{x}, y\}$ & $\{\hat{x}\}$ & $\{\hat{x}\}$ \\
\hline$\underline{\{x, \hat{x}, y\}}$ & $\underline{\{y\}}$ & $\underline{\{x, \hat{x}\}}$ \\
\hline
\end{tabular}

Table 1: Example 1

$\{y\} \neq\{x, y\}=C_{f}(\{x, \hat{x}, y\} \backslash\{\hat{x}\})$. So $C_{f}$ is not observably substitutable.

We will prove that if a choice function has a substitutable completion that satisfies IRC, it must satisfy observable IRC and NOVS. So it is weakly observably substitutable. Actually we will show that it is a corollary of a more general result. Since weakly observable substitutability (across workers) is sufficient for stable outcomes to exist, inspired by the substitutable completion idea we conjecture that if each firm's choice function $C_{f}$ has a completion $\bar{C}_{f}$ which is weakly observably substitutable (across workers), then stable outcomes may also exist. Because $\bar{C}_{f}$ may not be unitary, we cannot apply Theorem 1 to prove this conjecture. However, we will prove that $\bar{C}_{f}$ and $C_{f}$ have the same set of observable offer processes, and for every observable offer process $\mathbf{x}, \bar{C}_{f}(\mathbf{x})=C_{f}(\mathbf{x})$. Then if $\bar{C}_{f}$ is weakly observably substitutable (across workers), $C_{f}$ must be also weakly observably substitutable (across workers). Then Theorem 1 implies that the conjecture is correct.

To prove the above result we first prove two lemmas.

Lemma 1. Let $\bar{C}_{f}$ be a completion of a firm's choice function $C_{f}$. If $\bar{C}_{f}$ satisfies NSVS, then for all observable offer process $\boldsymbol{x}$ for $\bar{C}_{f}, \bar{C}_{f}(\boldsymbol{x})$ is feasible.

Proof. We prove it by contradiction. Suppose for some observable offer process $\mathbf{x}=$ $\left(x^{1}, \cdots, x^{M}\right)$ for $\bar{C}_{f}, \bar{C}_{f}(\mathbf{x})$ is not feasible. Then there exist two distinct contracts $x^{i}, x^{j} \in$ $\bar{C}_{f}(\mathbf{x})$ with $i<j$ such that $w_{x^{i}}=w_{x^{j}}$. Since $\mathbf{x}$ is observable, $x_{i} \in \bar{R}_{f}\left(\left\{x^{1}, \cdots, x^{j-1}\right\}\right)$. But $x_{i} \notin \bar{R}_{f}(\mathbf{x})$, so $x_{i} \in \bar{R}_{f}\left(\left\{x^{1}, \cdots, x^{j-1}\right\}\right) \backslash \bar{R}_{f}(\mathbf{x})$. Then since $\bar{C}_{f}$ satisfies NSVS, there exists $x_{k} \in \bar{C}_{f}\left(\left\{x^{1}, \cdots, x^{j-1}\right\}\right)$ such that $w_{x^{k}}=w_{x^{i}}=w_{x^{j}}$, which contradicts the observability of $\left(x^{1}, \cdots, x^{j}\right)$. So $\bar{C}_{f}(\mathbf{x})$ is feasible. 
Lemma 2. Let $\bar{C}_{f}$ be a completion of a firm's choice function $C_{f}$. If $\bar{C}_{f}$ satisfies NSVS, then $\bar{C}_{f}$ and $C_{f}$ have the same set of observable offer processes and for every observable offer process $\boldsymbol{x}, \bar{C}_{f}(\boldsymbol{x})=C_{f}(\boldsymbol{x})$.

Proof. First, for any observable offer process $\mathbf{x}$ for $\bar{C}_{f}$, by Lemma $1, \bar{C}_{f}(\mathbf{x})$ is feasible. So $C_{f}(\mathbf{x})=\bar{C}_{f}(\mathbf{x})$. This implies that $\mathbf{x}$ is also observable for $C_{f}$.

Second, for any observable offer process $\mathbf{x}=\left\{x^{1}, \cdots, x^{M}\right\}$ for $C_{f}$, we prove by induction that it is also observable for $\bar{C}_{f}$. It is obvious that $C_{f}\left(\left\{x^{1}\right\}\right)=\operatorname{bar} C_{f}\left(\left\{x^{1}\right\}\right)$ since $\bar{C}_{f}\left(\left\{x^{1}\right\}\right)$ must be feasible. Suppose $C_{f}\left(\left\{x^{1}, \cdots, x^{i}\right\}\right)=\tilde{C}_{f}\left(\left\{x^{1}, \cdots, x^{i}\right\}\right)$ for all $i \leq m<M$. Since $\left(x^{1}, \cdots, x^{m+1}\right)$ is observable for $C_{f}, w_{x^{m+1}} \notin C_{f}^{W}\left(\left\{x^{1}, \cdots, x^{m}\right\}\right)=$ $\bar{C}_{f}^{W}\left(\left\{x^{1}, \cdots, x^{m}\right\}\right)$. So $\left(x^{1}, \cdots, x^{m+1}\right)$ is also observable for $\bar{C}_{f}$. By Lemma $1 \tilde{C}_{f}\left(\left\{x^{1}, \cdots, x^{m+1}\right\}\right)$ is feasible. So $C_{f}\left(\left\{x^{1}, \cdots, x^{m+1}\right\}\right)=\bar{C}_{f}\left(\left\{x^{1}, \cdots, x^{m+1}\right\}\right)$. By induction $\mathbf{x}$ is observable for $\bar{C}_{f}$, and $C_{f}(\mathbf{x})=\tilde{C}_{f}(\mathbf{x})$.

Now we are ready to prove the main result in this section.

Proposition 1. A firm's choice function $C_{f}$ has a weakly observably substitutable (across workers) completion if and only if $C_{f}$ is weakly observably substitutable (across workers).

Proof. Let $\bar{C}_{f}$ be a completion of $C_{f}$ that is weakly observably substitutable (across workers). Then by Lemma 2 , for every observable offer process $\mathbf{x}$ for $C_{f}, \bar{C}_{f}(\mathbf{x})=C_{f}(\mathbf{x})$. Then if $\bar{C}_{f}$ satisfies NOVS, for any two observable offer processes $\mathbf{x}, \mathbf{x}^{\prime}$ for $f$ such that $\mathbf{x}^{\prime}$ is a subprocess of $\mathbf{x}, \bar{R}_{f}\left(\mathbf{x}^{\prime}\right) \backslash \bar{R}_{f}(\mathbf{x})=\emptyset$. This implies that $R_{f}\left(\mathbf{x}^{\prime}\right) \backslash R_{f}(\mathbf{x})=\emptyset$. So $C_{f}$ also satisfies NOVS.

If $\bar{C}_{f}$ satisfies NSVS, for any two observable offer processes $\mathbf{x}, \mathbf{x}^{\prime}$ for $C_{f}$ such that $\mathbf{x}^{\prime}$ is a subprocess of $\mathbf{x}$, if $\bar{R}_{f}\left(\mathbf{x}^{\prime}\right) \backslash \bar{R}_{f}(\mathbf{x}) \neq \emptyset$, for every $x \in \bar{R}_{f}\left(\mathbf{x}^{\prime}\right) \backslash \bar{R}_{f}(\mathbf{x})$ there exists $x^{\prime} \in \bar{C}_{f}\left(\mathbf{x}^{\prime}\right)$ such that $w_{x^{\prime}}=w_{x}$. Since $\bar{C}_{f}(\mathbf{x})=C_{f}(\mathbf{x})$ and $\bar{C}_{f}\left(\mathbf{x}^{\prime}\right)=C_{f}\left(\mathbf{x}^{\prime}\right), C_{f}$ must have the same property. So $C_{f}$ also satisfies NSVS.

For any observable offer process $\mathbf{x}$ for $C_{f}$ and any $Z \subseteq R_{f}(\mathbf{x})$, since $C_{f}(\mathbf{x})=\bar{C}_{f}(\mathbf{x})$, $\bar{C}_{f}(\mathbf{x})$ is feasible and $Z \subseteq \bar{R}_{f}(\mathbf{x})$. Since $\mathbf{x}$ is also observable for $\bar{C}_{f}$ and $\bar{C}_{f}$ satisfies observable IRC, $\bar{C}_{f}(\mathbf{x})=\bar{C}_{f}(\mathbf{x} \backslash Z)$. So $\bar{C}_{f}(\mathbf{x} \backslash Z)$ is also feasible, which implies that $\bar{C}_{f}(\mathbf{x} \backslash Z)=C_{f}(\mathbf{x} \backslash Z)$. Hence $C_{f}(\mathbf{x})=C_{f}(\mathbf{x} \backslash Z)$, which implies that $C_{f}$ satisfies observable IRC. So if $\bar{C}_{f}$ is weakly observably substitutable (across workers), $C_{f}$ must be also weakly observably substitutable (across workers). 
Of course if $C_{f}$ is weakly observably substitutable (across workers), it can be a completion of itself.

Since a substitutable completion that satisfies IRC is weakly observably substitutable, the following corollary is immediate.

Corollary 1. If a firm's choice function $C_{f}$ has a substitutable completion that satisfies IRC, $C_{f}$ is weakly observably substitutable.

\section{Unilateral Substitutability and IRC Imply Substi- tutable Completability}

In this section we show that if a choice function satisfies unilateral substitutability and IRC, it must has a substitutable completion.

Definition 7. A firm's choice function $C_{f}$ is unilaterally substitutable if for all $Z \subseteq X$ and all $x, z \in X \backslash Z$, if $z \in R_{f}(Z \cup\{z\})$ and $z \notin R_{f}(Z \cup\{x, z\})$, then there exists $z^{\prime} \in Z$ such that $w_{z}=w_{z^{\prime}}$.

Proposition 2. If a firm's choice function $C_{f}$ satisfies unilateral substitutability and IRC, then it is substitutably completable.

To prove the proposition we first prove a lemma

Lemma 3. If a firm's choice function $C_{f}$ satisfies unilateral substitutability and IRC, then for all $Z \subseteq X$ and all $x, z \in X \backslash Z$, if $z \in R_{f}(Z \cup\{z\})$ and $z \notin R_{f}(Z \cup\{x, z\})$, then there exists $\hat{z} \in C_{f}(Z \cup\{z\})$ such that $w_{\hat{z}}=w_{z}$.

Proof. We prove by contradiction. Let $w=w_{z}$. Suppose there does not exist $\hat{z} \in C_{f}(Z \cup$ $\{z\})$ such that $w_{\hat{z}}=w$, then it means that $Z_{w} \subseteq R_{f}(Z \cup\{z\})$. Since $z \in C_{f}(Z \cup\{x, z\})$ and $C_{f}$ is unitary, we also have $Z_{w} \subseteq R_{f}(Z \cup\{x, z\})$. Since $C_{f}$ satisfies IRC, $z \in R_{f}(Z \cup\{z\})$ and $z \notin R_{f}(Z \cup\{x, z\})$ imply that $z \in R_{f}\left(Z^{\prime} \cup\{z\}\right)$ and $z \notin R_{f}\left(Z^{\prime} \cup\{x, z\}\right)$ where $Z^{\prime}=Z \backslash Z_{w}$. However, by the unilateral substitutability of $C_{f}$ these imply that there exists $z^{\prime} \in Z^{\prime}$ such that $w_{z^{\prime}}=w$, which is a contradiction.

\section{Proof of Proposition 2:}


We construct a substitutable completion $\bar{C}_{f}$ of $C_{f}$. At step 0 below we let $\bar{C}_{f}(Z)=$ $C_{f}(Z)$ for all $Z \subseteq X_{f}$. Then there may exist multiple violations of substitutability in $\bar{C}_{f}$. At each following step we identify some violations of substitutability and correct them by updating $\bar{C}_{f}$. At the end of the procedure we obtain the choice function $\bar{C}_{f}$ which is substitutable.

- Step 0: Define $\bar{C}_{f}^{0}(Z) \equiv C_{f}(Z)$ for all $Z \subseteq X_{f}$. It is obvious that $\bar{C}_{f}^{0}$ satisfies unilateral substitutability and IRC.

- Step 1: We consider all $Z \varsubsetneqq X_{f}$ such that $\bar{R}_{f}^{0}(Z) \backslash \bar{R}_{f}^{0}\left(X_{f}\right) \neq \emptyset$. Lemma 3 says that for any $z \in \bar{R}_{f}^{0}(Z) \backslash \bar{R}_{f}^{0}\left(X_{f}\right)$ there exists $z^{\prime} \in \bar{C}_{f}^{0}(Z)$ such that $w_{z^{\prime}}=w_{z}$. So $\bar{C}_{f}^{0}(Z) \cup$ $\left(\bar{R}_{f}^{0}(Z) \backslash \bar{R}_{f}^{0}\left(X_{f}\right)\right)$ is not feasible. Then we define $\bar{C}_{f}^{1}(Z) \equiv \bar{C}_{f}^{0}(Z) \cup\left(\bar{R}_{f}^{0}(Z) \backslash \bar{R}_{f}^{0}\left(X_{f}\right)\right)$. For all other $Z$, define $\bar{C}_{f}^{1}(Z) \equiv \bar{C}_{f}^{0}(Z)$.

By this definition there is no violation of substitutability associated with $X_{f}$ in $\bar{C}_{f}^{1}$.

- Step $k \geq 2$ : We consider all $Y \varsubsetneqq X_{f}$ such that $|Y|=\left|X_{f}\right|-k+1$ and all $Z \varsubsetneqq Y$ such that $\bar{R}_{f}^{k-1}(Z) \backslash \bar{R}_{f}^{k-1}(Y) \neq \emptyset$. By our construction $\bar{C}_{f}^{k-1}$ always chooses weakly more contracts than $\bar{C}_{f}^{k^{\prime}-1}$ from the same set of contracts if $k>k^{\prime}$. So for any $z \in \bar{R}_{f}^{k-1}(Z) \backslash \bar{R}_{f}^{k-1}(Y), z \in \bar{R}_{f}^{k-1}(Z)$ implies that $z \in \bar{R}_{f}^{0}(Z)=R_{f}(Z)$. Now we prove that $z \notin R_{f}(Y)$.

Suppose the contrary that $z \in R_{f}(Y)$. Then $z \notin \bar{R}_{f}^{k-1}(Y)$ implies that at some earlier step $k^{\prime}$ we have $z \in \bar{R}_{f}^{k^{\prime}-1}(Y)$ but $z \notin \bar{R}_{f}^{k^{\prime}-1}(\hat{Y})$ for some $Y \varsubsetneqq \hat{Y} \subseteq X_{f}$ so that we define $\bar{C}_{f}^{k^{\prime}}(Y) \equiv \bar{C}_{f}^{k^{\prime}-1}(Y) \cup\left(\bar{R}_{f}^{k^{\prime}-1}(Y) \backslash \bar{R}_{f}^{k^{\prime}-1}(\hat{Y})\right)$. However, at step $k^{\prime}$ we should also have $z \in \bar{R}_{f}^{k^{\prime}-1}(Z)$ since $z \in \bar{R}_{f}^{k-1}(Z)$ and $k^{\prime}<k$. Then $Z \varsubsetneqq \hat{Y}$ and $\bar{R}_{f}^{k^{\prime}-1}(Z) \backslash \bar{R}_{f}^{k^{\prime}-1}(\hat{Y}) \neq \emptyset$ imply that at step $k^{\prime}$ we must also define $\bar{C}_{f}^{k^{\prime}}(Z) \equiv$ $\bar{C}_{f}^{k^{\prime}-1}(Z) \cup\left(\bar{R}_{f}^{k^{\prime}-1}(Z) \backslash \bar{R}_{f}^{k^{\prime}-1}(\hat{Y})\right)$. This means that $z \in \bar{C}_{f}^{k^{\prime}}(Z)$, which contradicts $z \in \bar{R}_{f}^{k-1}(Z)$. So $z \notin R_{f}(Y)$.

Hence $\bar{R}_{f}^{k-1}(Z) \backslash \bar{R}_{f}^{k-1}(Y) \subseteq R_{f}(Z) \backslash R_{f}(Y) \neq \emptyset$. Then Lemma 3 says that for any $z \in \bar{R}_{f}^{k-1}(Z) \backslash \bar{R}_{f}^{k-1}(Y) \subseteq R_{f}(Z) \backslash R_{f}(Y)$, there exists $z^{\prime} \in C_{f}(Z)$ such that $w_{z^{\prime}}=w_{z}$. So $C_{f}(Z) \cup\left(\bar{R}_{f}^{k-1}(Z) \backslash \bar{R}_{f}^{k-1}(Y)\right)$ is not feasible. If $\bar{C}_{f}^{k-1}(Z)=C_{f}(Z)$, then $\bar{C}_{f}^{k-1}(Z) \cup\left(\bar{R}_{f}^{k-1}(Z) \backslash \bar{R}_{f}^{k-1}(Y)\right)$ is not feasible. If $\bar{C}_{f}^{k-1}(Z) \neq C_{f}(Z)$, by our construction $\bar{C}_{f}^{k-1}(Z)$ is already not feasible. So in any case $\bar{C}_{f}^{k-1}(Z) \cup\left(\bar{R}_{f}^{k-1}(Z) \backslash \bar{R}_{f}^{k-1}(Y)\right)$ is not feasible. Define $\bar{C}_{f}^{k}(Z) \equiv \bar{C}_{f}^{k-1}(Z) \cup\left(\bar{R}_{f}^{k-1}(Z) \backslash \bar{R}_{f}^{k-1}(Y)\right)$. For all other $Z$, define $\bar{C}_{f}^{k}(Z) \equiv \bar{C}_{f}^{k-1}(Z)$. 
By this definition there is no violation of substitutability associated with any $Y \varsubsetneqq$ $X_{f}$ such that $|Y| \geq\left|X_{f}\right|-k+1$ in $\bar{C}_{f}^{k}$.

When $k=\left|X_{f}\right|$, we stop the procedure. Then $\bar{C}_{f}^{\left|X_{f}\right|}$ is the choice function we want. Let $\bar{C}_{f} \equiv \bar{C}_{f}^{\left|X_{f}\right|} . \bar{C}_{f}$ is a completion of $C_{f}$ since from the above procedure we know that $\bar{C}_{f}(Z) \neq C_{f}(Z)$ only when $\bar{C}_{f}(Z)$ is not feasible. $\bar{C}_{f}$ is substitutable because we eliminate all violations of substitutability in the above procedure.

The choice function $\bar{C}_{f}$ we construct in the above proof may not satisfy IRC. However, since it is substitutable, by Lemma 2 we know that the choice function $C_{f}$ must satisfy NOVS. Since $C_{f}$ further satisfies IRC, $C_{f}$ is weakly observably substitutable.

\section{Discussion}

Besides the existence of stable outcomes, Hatfield et al. (2015) also prove that observable substitutability and other two conditions are sufficient for COM to be strategy-proof. In this note we relax $I R C$ to observable $I R C$. It is an open question that whether the strategy-proofness of COM still holds if $I R C$ is replaced by observable IRC in their proof.

In our definition of COM we arbitrarily choose an ordering of all workers according to which workers make offers. However, the outcome of COM is actually independent of this ordering if all firms' choice functions are weakly observably substitutable across workers. This fact can be proved in the entirely same way as the similar result of Hatfield et al.

Lastly, in Section 5 we construct a substitutable completion of a unilaterally substitutable choice function that satisfies IRC. However, the completion may not satisfy IRC. It is also an open question that whether there exists a substitutable completion that satisfies IRC, and if yes, how to construct it.

\section{References}

Aygün, O. And T. Sönmez (2013): "Matching with contracts: Comment," The American Economic Review, 103, 2050-2051.

Hatfield, J. W. And F. Kojima (2010): "Substitutes and stability for matching with contracts," Journal of Economic Theory, 145, 1704-1723. 
Hatfield, J. W. And S. D. Kominers (2016): "Hidden Substitutes," working paper.

Hatfield, J. W., S. D. Kominers, And A. Westkamp (2015): "Stability, StrategyProofness, and Cumulative Offer Mechanisms," working paper.

Hatfield, J. W. And P. R. Milgrom (2005): "Matching with contracts," American Economic Review, 913-935.

KADAm, S. V. (2014): "Unilateral Substitutability implies Substitutable completability in many-to-one matching with contracts," working paper.

Kominers, S. D. (2012): "On the correspondence of contracts to salaries in (many-tomany) matching," Games and Economic Behavior, 75, 984-989. 